\title{
Challenges of Translating Classical Tamil Poetry into French: The Tiruppavai as Example
}

VASUMATHI BADRINATHAN

\begin{abstract}
This article focuses on classical Tamil poetry, represented by the Tiruppavai of Andal. The article seeks to understand the challenges of translating from Tamil into French, of a poetry that is non-contemporary, and which communicates layered meanings relating to religion, metaphysics, spirituality, nature and simply, the self. While every literary translation poses difficulties, translating classical Tamil poetry comes with a set of challenges by way of being spatially, temporally, contextually and even to an extent, linguistically removed from the present. Which route should the translator take: Faithful reproduction, brave reinvention or a middle path? The corpus for this article is drawn from two translations in French of the Tiruppavai that are studied and compared in this perspective.
\end{abstract}

Keywords: Poetry, Tiruppavai, Tamil, French, Translation.

\section{Introduction}

The world of translation is replete with contemporary works of literature. Literary works in translation belonging to earlier periods, though not absent, find a negligible place in this scene apart from academic circles. The reasons for this could be many. The difficulties imposed by the cultural ethos and mysticism of India as a whole could be a challenge, coupled with its linguistic complexities. The lack of interest for poetry in readers could be another factor. The religiosity and spiritual element in classical poetry could be yet another reason which publishers fear may not resonate with contemporary readers and which makes publishing such translation difficult. This 
article relies on a corpus of two translations from Tamil into French of the Tiruppavai: one by the renowned Indologist and scholar, Jean Filliozat in 1972 and the other by this author in 2019. In the light of what is explained above, it is not surprising that there is a gap of forty-seven years between the two translations with no known translations of this work in French in the interim period to the best of our knowledge.

\section{Background of the Tiruppavai: Context and Content}

A chef-d'oeuvre of Tamil literature, the Tiruppavai is a gem in itself. It remains a centrepiece of Sri Vaishnava philosophy propounded by Ramanuja, which rests on devotion to Vishnu as the supreme god. Andal is the only female amongst the twelve Alwars of Tamil Nadu, poet-saint-philosophers of Tamil Nadu. In her incredible life as one passionately in love with Vishnu, she ultimately unites directly with him into the altar. The Alwars lived between the $6^{\text {th }}$ and $9^{\text {th }}$ centuries and their collective poetry of four thousand verses is known as the Nalayira Divya Prabandham. Each individual poem or verse is called a Pasuram. The Alwars led the Bhakti movement in Tamil Nadu and their poetry is hailed as Tamil Marai or the Tamil Vedas - representing the esoteric and philosophical values of the Vedas in a tongue commonly understood in the land.

The Tiruppavai is composed of thirty verses of eight lines each. Each octave is replete with a delightful simplicity that belies their profundity; the verses shine with literary and philosophical connotations. There are several commentaries on the philosophical content of the Tiruppavai. Though the Tiruppavai includes thirty different poems, they are gathered together in one single unit which flows cohesively from one poem to the other, like chapters of a novel. Therefore, one could read them individually or chronologically from start to end. In either case, they remain beautifully coherent. The land 
is Gokulam, of cowherds, milkmaids and Krishna. The young girls wish to perform the pavai nombu, a ritual for the prosperity of the land and their own lives. In every poem, the group of girls travels from house to house, waking up the sleeping damsels and preparing them for the ritualistic bath. Their final goal is to reach Krishna, which they do after appealing to the mansion guardian, his father, his consort and finally to Krishna himself. Acclaimed as poetry of the highest order of the Visistadvaita philosophy, the poems are one of 'awakening' at many levels. Within each poem dwells a world in itself, complete with its multisensorial imagery and poetic elements. The Tiruppavai is a song of the exterior, much like an Impressionist painting. It draws vivid pictures of nature, the countryside, fields, seasons, birds, water, colours, the sun, and of the moon, the planets, cowherds, milk, butter, yoghurt. It could not be more complete in its pastoral description appealing to all senses. It combines a simple language with poetic elegance and depth. There is even one poem in dialogue form.

The Tiruppavai is also characterised by a strong musical element. Coming from an oral tradition, it was meant to be sung, and still continues to hold its position of pride within the Carnatic music repertoire. It remains a much-in-use poetry, recited regularly by the devout of the Sri Vaishnava tradition that especially gains momentum in the month of Margali (the winter month of Margashirsha). While many classical and ancient works have fallen into oblivion, Andal has continued to hold her sway over translators especially in English even in recent times (Chabbria, Shankar 2015; Venkatesan, 2010).

\section{The Problematic of the Dual Complexities of Tamil \& French}

Having explained the backdrop of the Tiruppavai and its fundamental spiritual and philosophical character, these very 
elements do not make it any easier for the translator. The Tiruppavai takes us to the time of divinity, of a time when God walked with man. Hence, we are dealing with poetry of a different level where Andal seeks salvation and leads the reader towards this ultimate objective. Taking a text such as the Tiruppavai into a foreign language, in this case, French, it makes a doubly challenging. How do you maintain a safe balance without much gaping cultural fractures and still keep it acceptable to a Francophone reader of target language? The Tiruppavai as the source text (ST) is a poetic document replete with complexities for the translator. Not just linguistic, but context, time and space-driven challenges too. The Tiruppavai has come down since ages, intact in a fairly homogenized version through the oral tradition. For academic purposes, we refer to a 1988 edition (Krishnaswami Iyengar) of the Tiruppavai as ST. We shall consider some excerpts of translated text (TT) from both the versions in question in this article and analyse the same. All re-translations in English from French in this article are those of this author and are provided to facilitate understanding for readers not familiar with French language.

\section{Keeping Close to the Original}

Right from the inaugural poem, Filliozat announces his fidelity to the ST. He remains charmingly close to Tamil culture in his choice of 'Yacotai' (Filliozat 1972: 6) for Yashoda, (Yashodai ilanshingam - the metaphor of Yashoda's lion cub) adding the typically Tamil 'ai' suffix while referring to Yashoda, the 'ai' also communicating 'belonging to'. This translator has simply chosen 'Yashoda' for its universality in her TT, two variations, driven solely by the translators' choice.

Let us look at some words that occur frequently in the Tiruppavai. It is not impossible to translate words as 'nai (ghee), 'kuttuvilakku' (lamp) into French. Filliozat simply uses 
'beurre' (butter) for 'nai' (ghee or clarified butter) (Filliozat 1972: 2) and 'lampadaire' (lamp on a stand) (Filliozat 1972: 20) for kuttuvilakku. However, this translator maintains the source text word as the translation did not quite reflect the totality of the word in her eyes. Are we simply seeking equivalence of a semantic order or are we looking for communicating the essence of the poem? Some detailed explanation here would be in order. The kuttuvilakku, to anyone familiar and raised in Tamil land and culture would associate this lamp to a characteristic shape, design, even a certain grace with feminine tones, coming from Tamil artistry. A kuttuvilakku from Tamil Nadu is very different from the Kerala oil lamps for example. Merely translating kuttuvilakku into 'lamp' for this translator, did not sufficiently evoke the beauty of this lamp or its design which plays an important role in this poem which is by far the most sensuous and intimate of all the thirty poems. The kuttuvilakku lights up subtly and sensuously the bed chamber, where the divine couple lies in serenity. So much so, that Andal chooses to start the poem with kuttuvilakku. Here are the beginning verses of this poemPasuram 19 (Nalayira Divya Prabandham: 84)

குத்து விளக்கெரியக் கோட்டுக் கால் கட்டில் மேல் மெத்தென்ற பஞ்ச சயனத்தின் மேல் ஏறி

Kuttuvilak eriya kottuk kaal kattil mel Mettenra panja sayanathin mel eri

A la lueur du kuttuvilakku, allongé sur un lit moelleux aux pieds d'ivoire (Badrinathan, 2019 : 47).

This translator being of Tamil origin chooses therefore to maintain the source text word as seen above, with a footnote explanation.

Nevertheless, Filliozat is not forgetful of the usefulness of source language words and source text words. He uses source 
text words for proper names, for living beings and also for elements of nature, some examples being Narayana (Pasuram 1), Sarnga (Vishu's bow, Pasuram 4), 'madhavi' (type of creeper, Pasuram 18). This translator however is more liberal with use of source text words as for example Parai (drum, Pasuram 1), Chakra, Panchajanya, Saranga (disc, conch, bow - all names of Vishnu's arms, Pasuram 4), ambal (type of flower, pasuram 14), madhavi (type of creeper, Pasuram 18), kuttuvilakku (lamp, Pasuram 19), pallandu (chant of praise, Pasuram 26) and source culture words such as nombu (the special ritual, Pasuram 2), kasumalai (necklace of gold coins, Pasuram 7), tulsi ${ }^{8}$ (the sacred plant, Pasuram 10). This choice of source language words is justified by their emblematic names and the iconic role they play in Tamil culture even today. A kasumalai cannot have any other form - it is necessarily a necklace with gold coins, nombu is the traditional feminine ritual in vigour even to this day. Is the tulsi translatable or does it fit well in a translated equivalent with all the symbolism it is associated with? That is also why this translator prefers to keep the key word 'parai' instead of 'drum' as the parai, is a polysemic leitmotif in the Tiruppavai. It is at once the drum, the object and also symbol of deliverance; it is both the means and the end.

However, code mixing has been a conscious choice in both translations. It does not imply that the translator's despair for lack of words but use the strategy deliberately to enhance the quality of the TT and render it as close to the original texture as possible. A strategy that Venuti (1995) would not be unhappy about, as it sufficiently 'foreignises' translation.

\footnotetext{
${ }^{8}$ Although Tulsi is a Tamil word, துழாய் is the ancient Tamil term used in the poem to mean Tulsi.
} 


\section{Form versus Content}

The length of the lines in translating poetry is a question of concern. Each of Andal's poems is an octave. Filliozat remains loyal to the eight lines. This translator has extended it to ten lines, as French language does not comfortably allow ease of translation in brevity. While some poems lend themselves to be fitted into the eight-line sequence, many do not. In order to maintain uniformity, this translator chose to maintain the translation into French in ten-line stanzas. Filliozat, for his part, remains in the eight-line limit and numbers them verses, but his lines double up. For example, in Pasuram 14, this is how he extends line 2 of the poem (Filliozat 1972: 14)

செங்கழுநீர் வாய் நெகிழ்ந்து ஆம்பல் வாய் கூம்பின காண் Senkayuneer vai negindu ambal vai koombinak kaan Les corolles des lotus rouges se sont ouvertes et les corolles des lotus blancs se sont fermées. Regarde. The petals of the red lotus have bloomed and the petals of the white lotus have closed. Look.

Or

In Pasuram 2 (Filliozat 1972: 2).

வையத்து வாழ்வீர்காள் நாமும் நம் பாவைக்குச் செய்யும் கிரிசைகள் கேளீரோ பாற்கடலுள்

Vaiyathu vaiveergaal namum nam pavaikku(ch)

Seiyyum girisaigal keliro parkadalul

Vous qui vivez dans le monde, n'écouterez-vous pas

les actions que nous devons accomplir pour notre

vœu?- Sur l'océan du lait

You who live in this world, won't you listen to 
Vasumathi Badrinathan

the actions that we must accomplish for our

vow? - Upon the ocean of milk

\section{When Prose Meets Poetry}

In many instances, Filliozat adopts simplicity and straightforwardness. In Pasuram 26, Filliozat (1972: 26) translates thus.

மேலையார் செய்வனகள் வேண்டுவன கேட்டியேல்

Melayar seivanangal venduvana kettiyel

Si tu demandes les choses nécessaires aux actes des

personnes éminentes

If you ask for the things needed for the act from eminent persons

In Pasuram 11, Filliozat (1972: 12) willingly introduces a conjugated verb which lends it a solid prosaic quality.

Ils partent pour détruire la force des ennemis et combattent They set out to destroy the strength of the enemies and fight.

The ST however eschews this succession of verbs (Nalayira Divya Prabandham 1988: 82)

செற்றார் திறலழியச் சென்று செருச்செய்யும்

Settraar tiralayiya senru serucheyyum

This translator's version (Badrinathan $2019: 31$ )

Et leur bravoure à vaincre l'ennemi dans d'illustres guerres And their valour in triumphing over the enemy in illustrious wars

In this case, this translator remains close to the original text and attempts to retain the poetic value. Another example is Pasuram 5. For the line 'vaayinaal paadi' which conveys 'mouthing praises', Filliozat (1972: 6) once again remains with the literal translation. 
வாயினால் பாடி மனத்தினால் சிந்திக்கப்

Vaayinaal paadi manathinaal sindhikka(p)

Quand, chantant de la bouche nous pensons à Lui

par l'esprit

When, singing by mouth we think of Him

through our minds.

Here, the logic is evident, as like Andal, herself, Filliozat insists on the verbal chant and the simultaneous mental devotion that go hand in hand (mouth and mind). While in the other translation, the translator (Badrinathan 2019: 19) decides to forego this element of contrast by using the verb 'sing'.

Nous Lui chanterons des louanges

Nous graverons dans nos pensées Son souvenir

We shall sing his praises

We shall engrave in our thoughts His memories

In some instances, Filliozat (1972:12) permits a rupture of sentence which rings an odd tone semantically. Like in Pasuram 11.

Toutes les amies de l'entourage sont venues, dans ta Cour elles sont entrées et tandis qu'elles chantent... All neighbouring friends have come in your Courtyard they entered and while they sing...

Is the intention to remain close to Andal who resorts at times to similar constructions? For example, in Pasuram 3, Andal says (Nalayira Divya Prabandham 1988: 81).

தேங்காதே புக்கிருந்து சீர்த்த ம முலை பற்றி வாங்கக் குடம் நிறைக்கும் வள்ளல் பெரும் பசுக்கள் Thengaday pukkirandu seerta mulai patri Vanga kudam niraikkum vallal perum pasukkal 
Patri vaanga, refers to the act of milking and both words go together. Filliozat seems to imitate this model in the example mentioned supra.

In Pasuram 16, the girls entreat the door keeper to let them into the mansion of Nandagopa. Here is the literal translation of the ST (Nalayira Divya Prabandham 1988: 84).

வாயால் முன்னமுன்னம் மாற்றதே அம்மா நீ

Vaayaal munnamunnam maatrade amma nee With your mouth first, do not refuse, mother

It is common in Tamil to use the idiomatic phrase 'with your mouth' to mean 'to speak', 'to utter', and to 'have a tongue' to mean someone who talks but does not act. Filliozat prefers the literal.

Ne refuse pas d'abord de la bouche, ô Père

Do not refuse with your mouth, $O$ Father.

Interestingly, he even replaces 'Amma', or mother, with' père' or father. The door keeper being a male, was it that which prompted Filliozat to choose the masculine? His choice he explains is based on the explanation that 'amma' is a vocative of 'amman' - maternal uncle or father; here the guardian of the door (Filliozat 1972: 48).

Another example would be Pasuram 14 (Nalayira Divya Prabandham 1988: 83). Here is first, the literal translation in English.

எங்களை முன்னம் எழுப்புவான் வாய் பேசும் நங்காய் எழுந்திராய் நாணாதாய் நாவுடையாய் Engalai munnam ezhuppuvan vai pesum Nangai ezhundiraai, naanadai naavudayay Us you wished to first awaken, your mouth said Girl wake up! Do you only have a tongue 
Filliozat (1972: 14)

Toi qui disait de bouche que tu nous éveillerais la première,

O notre sour, éveille-toi. Tu n'as pas honte? Toi qui [n'] as [que] la langue

You who said with your mouth that you would be the first to awaken us

O sister of ours, wake up. Aren't you ashamed? You, who only has a tongue?

This translator (Badrinathan 2019: 37)

Toi qui te vantais hier d'être la première à nous réveiller, Lève-toi donc! Tes promesses sont trompeuses

You who boasted yesterday of being the first one to wake us up Wake up yourself, won't you! Your promises are a betrayal

A scholarly, veteran Indologist and translator, it is evident that Filliozat did not make these choices arbitrarily. Through his detailed notes, commentary and analysis of the Tiruppavai, one can conjecture that Filliozat wished to be clear, concise, and conventional in expression when communicating the intentions of Andal to a typical reader of the French translation who is geographically and culturally distant from the ST. The thrust of the translation is distinctly on the concerns of correct communication and scholarship, rather than a preoccupation with its poetic elegance.

\section{Poetic Characteristics of the Tiruppavai}

Andal's construction of her verses is invested with musicality. Let us see how this offers itself to translation. The famous Pasuram 4 (Nalayira Divya Prabandham 1988: 81) paints images of rain, thunder and colour. The musicality is further enhanced with the richly alliterative and assonantal verses. This poem, Aazhi mazhai kanna, is anaphoric in its use of ayi 
and assonantal in its use of ya/zha (ழ்) the singular phonetic sound of Tamil.

ஆழி மழைக் கண்ணா ஒன்று நீ கை கரவேல்

ஆழியுள் புக்கு முகந்துகொடு ஆர்த்தேறி

ஊழி முதல்வன் உருவம் போல் மெய்கறுத்துப்

பாழியன் தொளுடைப் பற்பநாபன் கையில்

ஆழிபோல் மின்னி வலம்புரிபோல் நின்றதிர்ந்து

தாழாதே சர்ங்கம் உதைத்த சரமழைபோல்

வாழ உலகினில் பெய்திடாய் நாங்களும்

மார்கழி நீராட மகிழ்ந்தேலோரெம்பாவாய்

Ayimayai kanna onru nee kai kararavel

Ayiyul pukku mugundu kodu aarteri

Ooyi mudalvan uruvam pol mei karuttu

Payiyan toludaya Padmanabhan kaiyil

Ayi pol minni valampuri pol ninradirndu

Tayade Sarnga mudaitha sharamayai pol

Vaya ulaginil peindidai nangalum

Margali neerada magindelor empavai

To counter the impossibility of maintaining these poetic devices, this translator (Badrinathan 2019: 17) uses verb endings in the TT. The imperative in French, ending with the 'ez' ( pronounced [e] ) gives the translation a semblance of the power that is vested in the ST.

Plongez dans les profondeurs des océans,

Puisez pleinement, résonnez ensuite dans les cieux en tonnerre,

Vêtissez-vous de noir, telle la cause première, 
Resplendissez en nuées d'éclairs

Rugissez fermement comme le Panchajanya

Déchaînez-vous sur la terre comme une pluie incessante

[de flèches décochées de son Saranga,

Dive into the depths of the oceans

Draw freely only to resonate again as thunder in the skies

Dress up in dark hues, like the primordial cause

Radiate in lightening streaks

Roar firmly like the Panchajanya

Unleash yourself upon the earth

Like an unstoppable rain of arrows released by his Saranga

Filliozat (1972: 4) however, goes straight to the point. His use of the second person singular, the familiar 'tu' (you), reveals a disarming familiarity with the main character of the poem and also thereby investing the translation with a rhythmic energy.

$O$ toi dont les yeux font une pluie d'océan, ne cache rien dans ta main

Entre dans l'océan, puise, élève-toi en tonnant,

Devenu noir de corps à l'image du Premier Etre des âges,

Oh you whose eyes are an ocean of rain, hide nothing in your hand

Enter into the ocean, draw from it, arise thundering

Having become darkened in body like the Primordial Being of all ages 
In Pasuram 11 (Nalayira Divya Prabandham 1988: 82), Andal plays on the repetition of the consonne 'tra' at the beginning of each verse.

கற்றுக் கறவைக்

செற்றார் திறல்

குற்றம் ஓன்றில்லாத

புற்றரவல்குல்

சுற்றத்துத் தோழிமார்

முற்றம் புகுந்து

சிற்றாதே பேசாதே

எற்றுக்கு உறங்கும்

Kattru kkaravai

Setrar tiral

Kuttram onru illade

Puttru aravalgul

Sutrattu tozhimar

Muttram pugundu

Sitraday pesaday

Etrukku urangum

The clever use of ' $t+r a$ ' (the hard consonant $\mathrm{D}$ ) in Tamil gives to the texture of the verses, a 'crispy' effect, which corresponds to Andal's sharp injunctions to the lazy, sleeping girl in the story, exhorting her to rise and accompany her friends to the bathing ritual of the nombu ceremony. Neither Filliozat nor this translator has reproduced the effect of the Tamil ' $t+r a$ '. In Pasuram 19, less alliterations around ' $k$ ' and ' $t$ ', suggest the warmth and tenderness of the bed chamber, the 
beautiful breasts of Nappinnai, consort of Krishna, lying on the comfortable bed.

The first four verses are given below. Filliozat (1972: 20) translates as follows:

குத்து விளக்கெரியக் கோட்டுக் கால் கட்டில் மேல் மெத்தென்ற பஞ்ச சயனத்தி ம் ம மே ஏறி கொத்தலர் பூங்குழல் நப்பின்னை கொங்கை மேல் வைத்துக் கிடந்த மலர் மார்பா வாய் திறவாய்

Kuttuvillak eriya kottukkal kattil mel

Meddenra pancha sayanathin mel eri

Kothalar poonkuyal Nappinnai kongai mel

Vaithuk kidanda malar maarba vai tiravai

Tandis que brûle le lampadaire, sur le lit aux pieds

d'ivoire,

Reposant sur une douce couche aux cinq [qualités],

Le sein de NappiNNai à la chevelure aux fleurs

épanouies en bouquets

Posé sur ta poitrine largement étendue, ô toi, ouvre

la bouche!

While the lamp burns, on the bed with ivory legs

Resting on a soft couch having five qualities

The breast of Nappinnai's who wears on her hair blossomed flowers

Reclining on your large chest spread out, oh you, open your mouth! 
Filliozat's play of contrast of the French phonetic sounds- 'oo' $[\mathrm{u}]$ and ' $\mathrm{u}$ ' [y], give the verse the warmth and lilt of the ST.

This translator (Badrinathan 2019: 47) uses the consonant 'l' to achieve the same effect.

A la lueur du kuttuvilakku, allongé sur un lit moelleux aux pieds d'ivoire

Tu te reposes, Ta large poitrine étendue

Comme une fleur épanouie sur les seins de Nappinnai

Aux cheveux embellis de grappes de fleurs écloses

In the glow of the kuttuvilakku, reclining on a soft bed with ivory legs

You rest, Your broad chest spread out

Like a blossomed flower on Nappinnai's breast

She who wears bunches of bloom in her hair

Neither of the above two translations have however captured Andal's generous juxtaposition of ' $\mathrm{k}$ '.

Andal uses an astonishing range of poetic techniques. In Pasuram 24 (Nalayira Divya Prabandham 1988: 85), a panegyric verse, six of the eight verses ends with Potri (praise be to) (adi potri, tiral potri, pugazh potri, kazhal potri, gunam potri, vel potri) lending it a rich flavour of incantation. Both Filliozat and this translator have used 'toi qui' (you who) in repetition; Filliozat uses 'hommage' (praise) frequently. This translator, in addition to 'gloire' (glory/praise be) in repetition, also uses the past participle of the verbs to maintain the effect of litany.

அன்று இவ் உலகம் அளந்தாய் அடி போற்றி

சென்றங்குத் தென்இலங்கை செற்றாய் திறல் போற்றி 


\section{பொன்றச் சகடம் உதைத்தாய் புகழ் போற்றி}

Anru ivvulagam alaindai adi potri

Senrengudh tenni Lankai setraai tiral potri

Pondra chakatam udaithai pugal potri

This translator (Badrinathan 2019 : 56)

Toi qui autrefois as enjambé l'univers, gloire à Tes pieds!

Toi qui as triomphé dans le sud, au Lanka, gloire à Ta valeur!

Toi qui as écrasé la roue d'un coup de pied, gloire à Ta renommée!

You who once measured the universe, glory be to Thy feet!

You who triumphed in the south in Lanka, glory be to Thy valour!

You who crushed the wheel in one kick, glory be Thy fame!

While this option was relatively easier, other poems pose some veritable challenges. Note the softness created by the repetitive ' 1 ' in the entire $26^{\text {th }}$ pasuram- Maale, melaiyar, nyalathai, paalanna, polvana, saala, kola, aalinilaya. Is it even possible to truly reflect this stylistic impact in translation? Neither Filliozat nor this translator could do justice to this element in their translations. The beauty of such syllables can only be perceived, read and felt in the ST. Same is the case of the closing poem which plays on the sound 'nga' in every first word.

\section{Orality in Translation}

How does one convey a verse that was never meant to be written but rather sung, recited, communicated as was the Tiruppavai? In order to conserve and emphasise the oral nature of the poetry, this translator opts for inversing the order of the 
verses whereas Filliozat maintains the source word itself in the translation as in Pasuram 7.

கீசு கீசென்று எங்கும் ஆனைச் சாத்தன் கலந்து பேசின பேச்சரவம் கேட்டிலையோ பேய்ப் பெண்ணே

Keechu Keechu enru engum anai chathan kalandu

Pesina pecharavam kayttilayo pei penney?

"Keechu keechu” the anaichathan birds all of them together

Their talks don't you hear mad girl?

This translator (Badrinathan 2019 : 23)

O toi insensée! Engourdie comme une fille possédée, N'entends-tu pas le verbiage incohérent des oiseaux en réunion?

Oh you, senseless one! Benumbed like a possessed girl

Don't you hear the incoherent chatter of birds that crowd together?

Filliozat (1972: 8):

" Kis-kis » font partout les mainates en se réunissant ;

N'entends-tu pas le bruit des propos qu'ils se

tiennent? Fille stupide.

Keechu-keechu say the birds getting together all over

Don't you hear the noise of the discourse they are holding?

Foolish girl!

"Keechu keechu" is an onomatopoeia reflecting the noisy chattering of the birds. In commonly spoken Tamil, it is also used to represent a shrill intonation. Andal's closeness to the spoken word reflects in Filliozat who maintains the 
onomatopoeia. However, adhering closely as he always has to the ST, he respects the same order of verses with a loyal translation.

Let us here draw attention to the distinction that Nida (1964) proposes between 'formal' and 'dynamic' equivalence in translation. The latter by nature is dynamic, allows for freedom of the translator which infers that the translator in his preoccupation of making sense to the receptor, makes suitable alterations to the source text. Formal equivalence, on the other hand can be interpreted as the no-nonsense kind, the logical one. Such a translation naturally by its intent, stresses on fidelity towards the source text. In both the translations in question in this article, the translators blend the formal and the dynamic, though Filliozat tends to lean towards the formal. The choice of 'formal' or 'dynamic' depends largely on the purpose of the translation. This brings us to the objective of the translation. Filliozat's translation is of an academic nature. This is evident in the study of examples analysed above. His translation is preceded by a seventeen-page introduction to the Tiruppavai followed by almost sixty pages of detailed notes of the pasurams and plates of temple frescoes of the representation of the Tiruppavai. Every line of each poem is numbered for easy reference. This leaves no doubt about the objective of the translation which is to bring it as faithfully as possible to the reader with a well-researched scholarly publication. The second translation, which is by this author, does not share the same preoccupation. It is meant to help the common reader of French savour the beauty of Bhakti poetry while retaining the ST's flavour in the most convincing manner possible. With an introduction by the translator and a note on the Tiruppavai by Sri Aurobindo, this translation offers a fresh look at the Tiruppavai after nearly five decades.

\section{Translating the Untranslatable}


The surface simplicity of the Tiruppavai belies the difficulties that unfold in translation as we have noted. Let us consider the last line of the octave that closes every poem of the thirty poems. 'Elor empavai' is the refrain that is systematically preceded by a different prefix in each of the poems, changing subtly its meaning each time and investing it with a different depth each time. Scholars have submitted that this refrain is polysemic in offering many shades of symbolism. In this case, Filliozat settles for 'prends en considération notre vœu' (consider our vow). This translator chooses 'Notre prière unique en sera fructueuse' (Our unique prayer will thus be fruitful), achieving an alexandrine verse. It seems virtually impossible to translate this clever line which changes in hue in every poem, depending on its preceding words (arulelor empavai, maghindélor empavai, kulirndélor empavai and so on) making it a translator's riddle.

Translating Tiruppavai throws up many questions, often unanswerable. How does one maintain the symbolic value of the Hindu 'bath' which is fundamental in the nombu ritual of the Tiruppavai- 'neeradal', or bath, is layered in many meanings, including sexual bliss? How does one transfer the typical Tamil or pan-Indian expression 'at the feet of', to underline blessing, surrender to a higher order and not servitude? Or yet how does one communicate the Hindu symbolism relating to the different parts of the day, like dawn? How does one attribute to 'little parrot', 'idiotic girl', a connotation of affection? Or hold the lotus as the flower of beauty, of divinity? Or the idiomatic expression 'you only have a tongue', alluding to one who speaks a lot but does not act? Or the paronomasia communicated by Andal through the word 'oruthi' (Pasuram 25) which signifies both 'she' and 'the other' (referring to Krishna's biological mother and foster mother) at the same time. What a puzzle Andal conveys gently in a woman-led set of poems! The Tiruppavai is a veritable 
cultural conundrum for the translator. The translator has to weave through the verses, much like the cowherd girls themselves, each undertaking their journey towards their final destination.

Both translations herein discussed the Tiruppavai, though very different, share some striking similarities. Neither is very adventurous nor contemporary in its approach. There are no neologisms or out-of-the box interpretations. In this respect, both translations remain loyal to the ST, though in different ways. But gaps as compared to the ST are a given and do appear in both the translations, which Venuti (1995) confirms are the very essence of translation between two languageslooking for similarities where dissimilarities abound. Moreover, to refer to the oft-used expression, traduttore traditore - translation is betrayal. But we can once again take recourse to Nida (1964) who clarifies that a complete exactitude cannot be achieved in translation. Mimicking would lead to disastrous results. So, we translate what we feel comes closest, sounds logical and thereby remain "safe".

\section{Conclusion}

It is evident that much as poetry is an individual and solitary activity. Translation is one too. It stems from a subjective interpretation of the poem, of the words, of the universe represented therein. It is by no means a dialectical position between the author on one side and the translator on the other. Translation is as much a work of art for the translator as it is for the author and as Spivak (1993: 183) expresses, "it is the most intimate part of reading". The original work passes through the filter of the translator's sensibilities in order to convert itself into another text. In that sense, a translation is an art of creativity, of approximation, of interpretation. Hence, two translations will never be the same, and it would only be the better for it. Moreover, the translator is a mediator. In both 
the studied translations of the Tiruppavai in this article, the translators have essayed to build a bridge between the reader and the source text on a subject seemingly simple, but with culturally heavy concepts that on the one hand gives the freedom of interpretation in translation and on the other hand restrain it (Venuti 2013). We could say that the translator occupies the 'third space' that Bhabha (1994) envisaged, close to the source text, yet distant as it from one language to the other and creates a new translated text which lies in between, a new text that is retold centuries later to a new audience and still meaningful. It is as much an activity of interculturality as it as a to and fro between two texts in two languages and a world of alterity. This duality of the translated text is its very privilege. Both Filliozat and this translator situate themselves in this intercultural interlingual space. Having argued thus, it can be concluded that literary translation, and especially that of poetry belonging to another era, certainly poses challenges but they are a necessary hurdle to cross in the interest of literature and its wider reach through the medium of translation.

Note: All retranslations into English of the French translations are done by the author.

\section{References}

Badrinathan, VASUMATHI. 2019. Tiruppavai ou le chant matinal de Margali. Paris: Editions Banyan.

Bнавна, Hомі K. 1994. The Location of Culture. London \& New York: Routledge.

Filliozat, JeAN. 1972. Un texte tamoul de dévotion vishnouïte. Le Tiruppāvai d'Ānțāl. Pondichéry: Institut Français d'Indologie.

KRISHNASWAMI IYENGAR, S. (ed.). 1988. Nalayira Divya Prabandham. Mudalayiram. Puduvai: All India Press. 
Sarukkai ChHabria, Priya, and Shankar, Ravi. (trans.). 2015. Andal: The Autobiography of a Goddess. New Delhi: Zubaan.

SPIVAK, GAYATRI C. 1993. Outside in the Teaching Machine. London \& New York: Routledge.

Venkatesan, Archana. 2010. The Secret Garland: Antal's Tiruppavai and Nacciyar Tirumoli. New York: Oxford University Press.

Venuti, LAWRENCE. 1995. The Translator's Invisibility: A History of Translation. London \& New York: Routledge. VenUTI, LAWRENCE. 2013. Translation Changes Everything: Theory and practice. London \& New York: Routledge.

$$
\text { *** }
$$

Cite this work:

BADRINATHAN, VASUMATHI. 2020. Challenges of Translating Classical Tamil Poetry into French: The Tiruppavai as Example. Translation Today, Vol. 14(1). 77-99. DOI: 10.46623/tt/2020.14.1.ar6. 